\title{
Cultural Journeys in the Information Society
}

\author{
G. Fakas, A.Kakas \\ and, \\ D. Dionisiou, M. Dionisiou, A. Kentonis, K. Pattichis, A. Pitsillides and C.Schizas, \\ Department of Computer Science, University of Cyprus, Cyprus
}

\begin{abstract}
This paper describes the Cultural Journeys in the Information Society (CJIS) project. CJIS project is an INCO project (973324) funded by the ECC and its aims to address the problem of electronic information roads at various levels and to develop a prototype system of such roads. The prototype will concentrate at first on cultural and historical information and the social relevance of such roads in education and training. This paper defines the notion of electronic information roads and explores the technical form which these roads can take.
\end{abstract}

An electronic road is the user's navigation path through a multimedia environment. More precisely, it is a series of links to the system's Information Units (IU) the user chooses to follow through. IUs are the building blocks of the available information content and consist of the actual data (e.g. segment of video, image, sound or text) with an attached metadata index (semantic or system). The system produces a number of dynamic links that point the user to new Information Units. These dynamic links are based on the IU's semantic nature and the user's profile.

Index Terms-Electronic Roads, Information Navigation, Search Engine, Meta-data

\section{INTRODUCTION}

Over the last fow years there has been an explosion in the importance of research and development in multimedia systems and their deployment over the Internet and WWW. However, these systems usually have limited interconnections among them; they may be viewed as virtual territories with very few existing facilities that can help users explore and retrieve relevant information. They apply to discrete data spaces only, failing to provide users with a means of continuous exploration in the related information space that often spans multiple data spaces. As a result, uniformed users may be lost or retracted from their intended destinations if they can not describe a desired journey explicitly. Thus, there is a strong need to address this problem and to provide information guides that would allow a coherent exploration of information; an exploration that can be viewed as a structured journey in the available information.

Search engines are used to gather a list of potential interesting sites in an effort to solve this problem.
Additionally, human intervention for the update of interconnections is often employed. Both approaches have major disadvantages. The required time and effort for the manual update is both overwhelming and expensive while search engines often respond with a large set of sites that are only peripherally related to the actual query. A great amount of research is being invested towards the creation of more powerful and intelligent search engines.

Electronic Roads can be thought of as the underlying fabric of the space of information and the Information Society at large. They shape this space and define a novel way of acquiring, using and exchanging information which ultimately can result in new social links between those who use these roads.

An Electronic Road creates an information map with spatial, temporal and context or more generally semantic associations amongst different information elements. These electronic roads could be based on existing physical roads mirroring the information available in the physical area of the road or they could be completely thematic roads that traverse information that is semantically related but of different nature at different locations and time. Users who are interested in a specific topic would search for and take an appropriate Electronic Road. This will provide them with a traversal of a meaningful set of related information sites with each different user selecting a journey that suits their own interests and priorities in a dynamic way as they follow the road and discover new information that they judge useful. The user therefore is allowed or invited to explore the available information by dynamically selecting and forming a personalized information journey that suits their own needs and interests.

A central challenging issue in the formation of these information roads is to define what indeed is the appropriate way to link, structure and organize information together. The available information needs to be structured in a way that supports flexible learners, rather than experts. For this reason every piece of available information in the system is associated with some metadata (semantic and system). Metadata can be maintained according to several standards such as the Dublin Core[9], XML[3] and RDF[6].

The primary requirement of an Electronic Road is to achieve a network of information in which the traveler can: 
1. explore the information space in a natural way where the information offered remains continuously interesting (the system will provide dynamic links),

2. adapt his/her exploration mode according to personal preferences both at the start and during the journey (the system will maintain a user profile),

3. learn new information, particularly new relevant associations of her/his original domain of interest with other domains,

4. offer as well as take information along his/her route, (the system will record his/her journeys),

In order to address the above mentioned requirements a number of specific research problems need to be addressed:

1. Develop adaptive and intelligent presentation multimedia systems.

2. Build digital libraries for heterogeneous types of data and support sophisticated retrieval mechanisms $[5,2$, 1].

3. Examine the potential of state-of-the-art network technology (e.g. ATM, ISDN, new Internet architectures such as DiffServ ) and design reliable and effective networking algorithms [7]. Also refine search results based on current network and its state.

4. Design and implement a metacomputing environment for amalgamating into one entity all the resources comprising an electronic road system.

\section{CULTURAL JOURNEYS}

An electronic road is the user's navigation path through a multimedia environment. It is a series of links to the system's Information Units (IU) the user chooses to follow through. IUs are the building blocks of the system and consist of the actual data (e.g. segment of video, image,

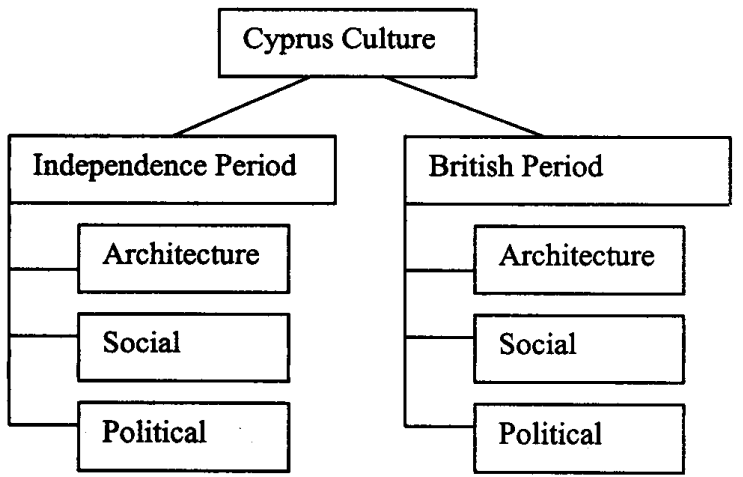

Fig. 1 Semantic Dictionary

sound or text) with an attached metadata index (semantic and system). The system produces a number of dynamic links that point the user to new Information Units. These dynamic links are based on the IU semantic dictionary. This semantic dictionary has a hierarchical structure from general to specific. For instance, figure 1 shows a (small part of the) semantic dictionary for Cyprus Culture.

\section{A. CJIS Concepts}

We have the semantic dictionary (described above) and the digital library of the IUs holding the content.

A secondary structure is that of an Information Element (IE) or NodeID which consists of a tuple of Information Units (IU) on the same subject. An IE is the total information /content in the different media that could be composed together to be presented to the user.

As mentioned above, the building block of the system is the IU, which consists of the actual data (e.g. segment of video, image, sound or text) with an attached metadata index as depicted in the following table.

\begin{tabular}{|c|c|}
\hline ation 2 & (semantic and orstem) WCet \\
\hline IUID & An identification of the IU \\
\hline NodeId & $\begin{array}{l}\text { An identification of the information elements } \\
\text { or nodes this can belong to }\end{array}$ \\
\hline $\begin{array}{l}\text { Semantic } \\
\text { Keywords }\end{array}$ & $\begin{array}{l}\text { The semantic dictionary nodes the IU } \\
\text { belongs to. } \\
\text { e.g. Cyprus Culture/Independence Period }\end{array}$ \\
\hline User Group & $\begin{array}{l}\text { The group of users that might be interested in } \\
\text { this IU. Users are classified by Age (12-18 } \\
\text { and older than } 18) \text { and Depth of Interest } \\
\text { (Low, Medium, High) }\end{array}$ \\
\hline Format & i.e. text; image, video \\
\hline System Format & $\begin{array}{l}\text { It provides information about the format of } \\
\text { resource which could be used to better utilise } \\
\text { the networks resources. E.g. if the resource is. } \\
\text { a video segment it could provide the bit rate. }\end{array}$ \\
\hline Language & $\begin{array}{l}\text { the language the document is written e.g. } \\
\text { English, Greek }\end{array}$ \\
\hline District & Cyprus.Kerynia, Cyprus.Famagusta \\
\hline Period/Time & e.g. pre-historic \\
\hline Other keywords & e.g. Zenon: \\
\hline
\end{tabular}

The system also maintains a profile about the user's initial preferences, records semantic metadata for every user's stops and proposes a User Profile Record. The User Profiler consists of three main sub-modules:

1. Initial User Profiler i.e. general information about the user and his/her preferences

2. Recorded User Profiler. recorded user's behavior i.e. User Previous stops.

3. ProposeUserProfile. The system takes into consideration the information in 1 and 2 and returns a proposed User Profile record.

The Presentation Unit (PU) is what the user sees. It presents an element of information (also called a node). Figure 2 shows a typical PU. This has the following general structure: 
1. Static Links. These are links to the Semantic Dictionary of Context in a tree structure.

2. Dynamic Links. The system produces a dynamic list of links according to user profile, and current IU metadata.

- Primary links give extra information in the primary (if any) interests of the user (relatively to the current position on the semantic dictionary).

- Lateral links change context to a "related" context.

3. User Profile. The user can define (and redefine) his/her preferences and interests.

4. Information Units:

- Text. It contains textual content (of the current position's node information element).

- Image. It contains an image content (of the current position's node information element).

- Video. It contains a video (of the current position's node information element).

Also the user is able to search for information, save a journey or recall an old journey.

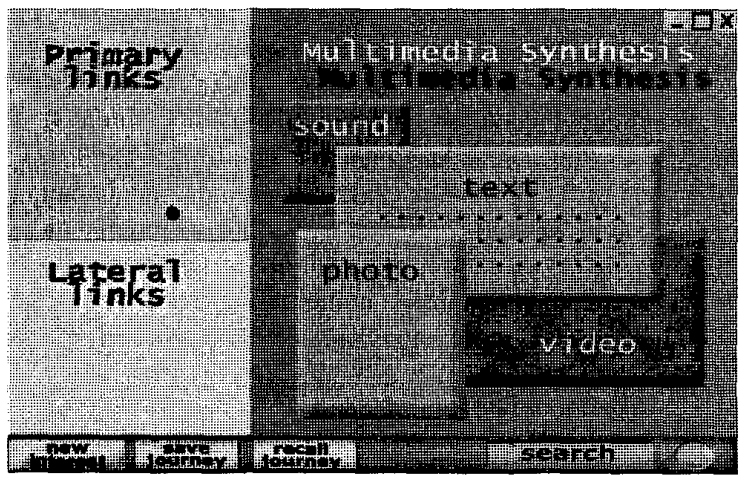

Fig. 2, The Presentation Unit

\section{B. Electronic roads Functionality}

Every time the user chooses a dynamic link the following actions are taken by the system.

1. User profiler. This module maintains a profile about the user from her/his initial preferences and records of the semantic metadata of the user's previous stops and proposes a User Profile Record. The user's profiler is based on the Proposal For An Open Profiling Standard [4].

2. IUs Selector. This module takes as input the user's next choice's nodeID and returns 3 ranked lists; one for each IU type. From these the system will select a subgroup in order to find the next presentation unit.

3. System Based Refiner (IU). The delivery of information via a network needs to meet certain Quality of Service (QoS) requirements dictated by the user application [8].The System Refiner considers the lists of IUs, the available bandwidth and the QoS requirements and rearranges or modifies the list. As a result of the actions of System Refiner some information units might be omitted or have their rank changed.

4. Link generator. This module takes as input the current's position metadata and user's profile and produces a list of related links to new nodes (information elements). These links could be either primary or lateral.

5. System Based Refiner (Link). The output of Link generator is a list of links. This module considers the list of links, the bandwidth and QoS requirements and rearranges the ranking of the list. As a result of the actions of System Refiner some links might be omitted.

6. PUs/Page Composer. This module takes as input the outputs of the System Refiner and Link Generator and produces the final Web document that the user will see. The system delivers a multi-frame document. The document frameset is dynamic i.e. one or more Information Unit frames might be omitted or rearranged.

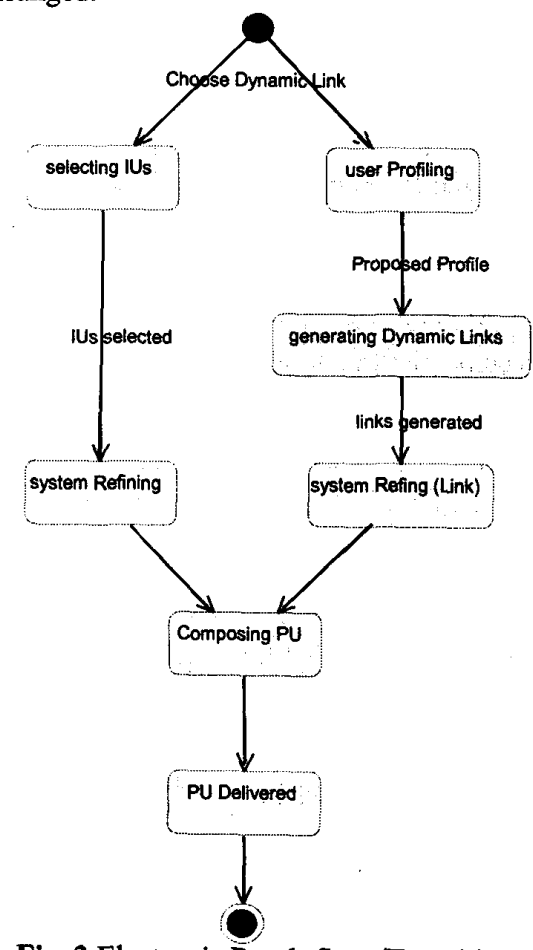

Fig. 3 Electronic Roads State/Transitions

\section{A CJIS Scenario}

In general, the system produces and presents to the user links to Information Elements (IEs). The user chooses at each point a link and this forms a road. The journey in figure 4 is based on the Semantic Dictionary presented in figure 1 .

The roads, the system produces are initially based on the Semantic Dictionary (SD). Assuming, we are starting a journey from the root of the SD (i.e. Cyprus Culture) the 
system will display information from the associated IE and links to other IEs whose Semantic Keywords (see metadata) are associated with that node of the SD. The primary links to this point will be Independence Period and British Period. If the user selects the Independence Period road, then again the system will display the associated IE to this node. Primary links of this will be Architecture, Social and Political, and Lateral links will be British Period. Similarly, if the user chooses Political, then the new PU will have primary and lateral links as shown below.

The above scenario was kept very simple, in order to demonstrate how primary and lateral links are produced and did not take into consideration other parameters that contribute to links dynamism, such as user profile and system metadata.

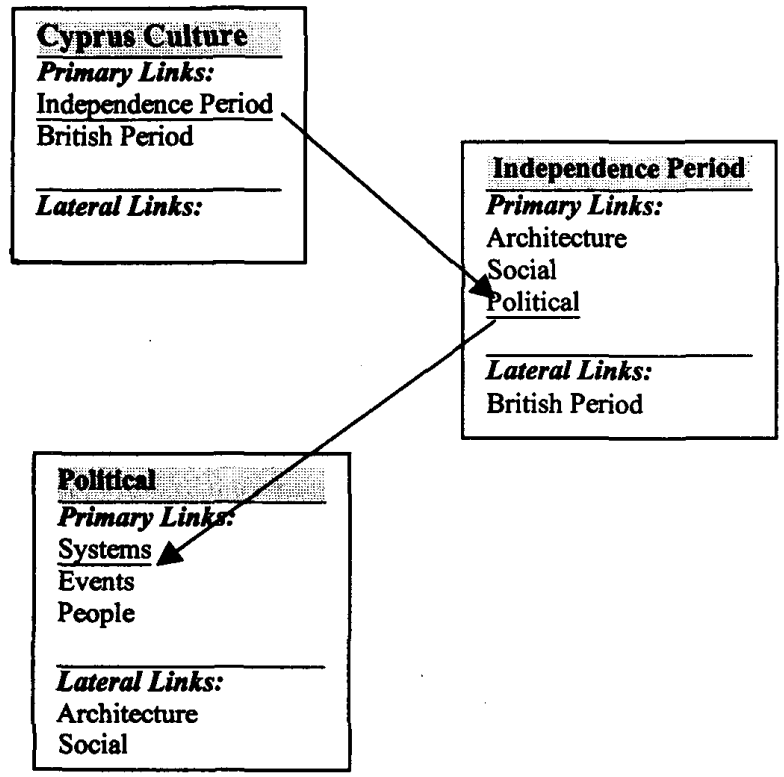

Fig. 4 A Cultural Journey

\section{IMPLEMENTATION ARCHITECTURE OF CJIS}

The software platforms that we will use are Java, JavaScript, Java RMI, JDBC, SQL DBMS, Dublin Core and hardware platform is Windows NT.

1. User Interface Agent. It will run through a web browser and will consist of two frames:

- Frame 1: Electronic Roads. This is a Java Applet that will get user's input and interface with the main ER system that will be running on the server.

- Frame 2: IU Content. This is the IU in an HTML format loaded from a URL address.

2. Server Site. This is a Java Application. This is where the ER functionality is encapsulated. It is responsible to respond to the users. It also access the Database Management System. Use RMI and JDBC.
3. IU and Metadata storage and retrieval. IUs will be stored as HTML documents. IUs metadata will be stored in an SQL DBMS (e.g. Microsoft Access) according to Dublin Core standard [9]. JDBC will be used to access the metadata from the main server Java application.

4. Client Server Communication. Java RMI will used for the communication needs between the client and server. RMI is a simple and abstract but powerful enough for the communication needs for our system. Other communication alternatives are also investigated such as Mozart and MANIFOLD.

5. Hardware Platforms. The client site is platform independent. The server Java application will be implemented and run on Microsoft Windows NTs.

6. Network. We will consider an Internet and Internet architecture such as DiffServ, and ATM.

\section{CONCLUSIONS}

In conclusion, this paper describes the Cultural Journeys in the Information Society (CJIS) project. This project aims to address the problem of electronic information roads at various levels. The paper defines the notion of electronic information roads and explores the technical form which these roads can take.

\section{REFERENCES}

[1] J, Boyan, D. Freitag, and G. Joachims, A Machine Leaming Architecture For Optimizing Web Search Engines, Proc. AAAI Wksp. Internet Based Information System 1996

[2] S. Brin and L. Page, The anatomy of a large-scale hypertextual Web search engine, Proc. $7^{\text {th }}$ International WWW Conference, Australia, 1998

[3] Extensible Markup Language, W3C, available at http://www.w3.org/XML/

[4] P. Hesley et al., Proposal for an Open Profiling Standand, W3C, available at http://www.w3.org/TR/NOTE-OPS-FrameWork.htm

[5] S. Lawrence, and C. L. Giles, Searching the Web, General and Scientific Information Access, IEEE Communications Magazine, January, 1999

[6] E. Miller, An introduction to the resource description framework D-lib Magazine, May 1998. Also available at http://www.dlib.org/dlib/may98/miller/05miller.html

[7] A. Pitsilides et al., Effective Control of Traffic Flow in ATM Networks Using Fuzzy Explicit Rate Marking, IEEE Joumal on Selected Areas in Communications, Vol. 15, No. 2 February 1997

[8] Z. Wang and J., Crowfort, Quality-of-Service Routing for Supporting Multimedia Applications, IEEE Journal on Selected Areas in Communications, Vol. 24, No. 7 February 1996

[9] S. Weibel, R. Iannella, W. Cathro, The $4^{\text {th }}$ Dublin Core metadata workshop report. D-Lib Magazine, June 1997, also available at http://www.dlib.org/dlib/june97/metadata/06weibel.html.

\section{ACKNOWLEDGMENTS}

We would like to thank the project partners for their contribution: ISSR, Cairo, Egypt, RSS, Amman, Jordan, NTUA and InfoGroup Athens, Greece, and MIDE, Spain. 